\title{
Automated identification of retinopathy of prematurity by image-based deep learning
}

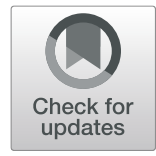

\author{
Yan Tong ${ }^{1}$, Wei Lu', Qin-qin Deng ${ }^{1}$, Changzheng Chen ${ }^{1}$ and Yin Shen ${ }^{1,2^{*}}$
}

\begin{abstract}
Background: Retinopathy of prematurity (ROP) is a leading cause of childhood blindness worldwide but can be a treatable retinal disease with appropriate and timely diagnosis. This study was performed to develop a robust intelligent system based on deep learning to automatically classify the severity of ROP from fundus images and detect the stage of ROP and presence of plus disease to enable automated diagnosis and further treatment.

Methods: A total of 36,231 fundus images were labeled by 13 licensed retinal experts. A 101-layer convolutional neural network (ResNet) and a faster region-based convolutional neural network (Faster-RCNN) were trained for image classification and identification. We applied a 10-fold cross-validation method to train and optimize our algorithms. The accuracy, sensitivity, and specificity were assessed in a four-degree classification task to evaluate the performance of the intelligent system. The performance of the system was compared with results obtained by two retinal experts. Moreover, the system was designed to detect the stage of ROP and presence of plus disease as well as to highlight lesion regions based on an object detection network using Faster-RCNN.

Results: The system achieved an accuracy of 0.903 for the ROP severity classification. Specifically, the accuracies in discriminating normal, mild, semi-urgent, and urgent were $0.883,0.900,0.957$, and 0.870 , respectively; the corresponding accuracies of the two experts were 0.902 and 0.898 . Furthermore, our model achieved an accuracy of 0.957 for detecting the stage of ROP and 0.896 for detecting plus disease; the accuracies in discriminating stage I to stage $V$ were $0.876,0.942,0.968,0.998$ and 0.999 , respectively.
\end{abstract}

Conclusions: Our system was able to detect ROP and differentiate four-level classification fundus images with high accuracy and specificity. The performance of the system was comparable to or better than that of human experts, demonstrating that this system could be used to support clinical decisions.

Keywords: Deep learning, Retinopathy of prematurity, Artificial intelligence, Fundus image

\section{Background}

Retinopathy of prematurity (ROP) is a proliferative retinal vascular disease that affects approximately twothirds of premature infants who weigh less than $1250 \mathrm{~g}$ at birth. It is associated with abnormal retinal vascular development at the boundary of vascularized and avascular peripheral retina $[1,2]$. Worldwide, an estimated 30,000 premature infants annually experience blindness

\footnotetext{
*Correspondence: yinshen@whu.edu.cn

${ }^{1}$ Eye Center, Renmin Hospital of Wuhan University, Wuhan 430060, Hubei, China

${ }^{2}$ Medical Research Institute, Wuhan University, Wuhan, Hubei, China
}

or severe loss of vision due to ROP [3]. Most cases of ROP are mild and resolve spontaneously without intervention; 5 to $10 \%$ of cases progress to more severe ROP, which can lead to retinal detachment or distortion of the retina and permanent blindness if left untreated [4]. Whereas clinical diagnosis and early disease detection remain subjective; high levels of inconsistency in ROP diagnosis have been observed even among ROP experts $[5,6]$. Therefore, it is urgent to establish a screening tool that can rapidly identify fundus images requiring further attention and critical analysis by ophthalmologists,

(c) The Author(s). 2020 Open Access This article is licensed under a Creative Commons Attribution 4.0 International License, which permits use, sharing, adaptation, distribution and reproduction in any medium or format, as long as you give appropriate credit to the original author(s) and the source, provide a link to the Creative Commons licence, and indicate if changes were made. The images or other third party material in this article are included in the article's Creative Commons licence, unless indicated otherwise in a credit line to the material. If material is not included in the article's Creative Commons licence and your intended use is not permitted by statutory regulation or exceeds the permitted use, you will need to obtain permission directly from the copyright holder. To view a copy of this licence, visit http://creativecommons.org/licenses/by/4.0/ The Creative Commons Public Domain Dedication waiver (http://creativecommons.org/publicdomain/zero/1.0/) applies to the data made available in this article, unless otherwise stated in a credit line to the data. 
thereby increasing the accuracy and efficiency of diagnosis.

Artificial intelligence (AI), inspired by the multilayered human neuronal system, has achieved great performance within medical imaging interpretation and triage tasks, allowing clinical experts to diagnose diseases efficiently and untrained technicians to objectively screen more patients. Deep learning (DL) has significantly extended the capabilities of images classification, object detection, drug discovery, and robot functions [7]. Convolutional neural networks (CNNs) are DL algorithms commonly applied in image classification, which have been successfully used in the diagnosis of skin cancer [8], lung cancer [9], glioma [10], and breast histopathology [11]. DL has achieved automated detection of retinal diseases [12, 13], including diabetic retinopathy [14], glaucoma [15], agerelated macular degeneration, and cataracts [16]. Recently, several studies regarding the diagnosis of ROP with AI have achieved promising results. Approaches for automated identification of plus disease in ROP depend on traditional approaches, such as machine learning with handcrafted features [17]. Gelman established a computer-based image analysis system to distinguish plus disease with 95\% accuracy, which is comparable with expert diagnosis [18]. Brown et al. developed an algorithm based on DL to automatically distinguish the presence of plus disease or pre-plus disease with high sensitivity and specificity [19]. However, the abovementioned studies were mainly focused on plus disease in ROP. An automated ROP diagnosis system that can analyze real-world clinical features (i.e., stage and zone of ROP as well as the presence of plus disease) is rare.

In this study, we established an intelligent system to achieve detection and classification of ROP in fundus images. The purpose of our study was to: (1) implement and evaluate a CNN-based DL system for four-level diagnosis (normal, mild, semi-urgent, urgent) of ROP in fundus images; (2) determine the accuracy of the system by comparing its diagnostic performance with that of experienced retinal experts; (3) detect the stage of ROP and presence of plus disease, and predict lesion location in fundus images using a faster region-based convolutional neural network (Faster-RCNN).

\section{Methods}

\section{Ethics approval}

Collection and labeling of fundus images were performed by ophthalmologists at Renmin Hospital of Wuhan University Eye Center. This study followed the tenets of the Declaration of Helsinki [19], and was approved by the institutional review board of Renmin Hospital of Wuhan University (ID: WDRY2019-K032). For all involved patients, written informed consent was obtained from their parents for imaging and study participation. In addition, we deleted all patients' sensitive information prior to image viewing, to ensure that their personal information remained anonymous and confidential.

\section{Data sets}

For algorithm development, a total of 38,895 fundus images from the ROP screening (from February 1, 2012, to October 1, 2016) were retrospectively collected from Renmin Hospital of Wuhan University Eye Center. All images were obtained using a wide-angle imaging device (RetCam; Clarity Medical Systems, Pleasanton, CA). The resolution of the image is $640 \times 480$ pixels. The dataset also included follow-up images from the same patients who underwent ROP screening.

\section{Image labeling, preprocessing and dataset division}

The overall experimental design and dataset selection process is shown in Fig. 1. The current study invited 13 licensed ophthalmologists, who specialized in retinal diseases diagnosis. Images were randomly assigned to 11 junior retinal experts for first-round screening and labeling. In the second round, the remaining two senior retinal experts who have over 10 years of individual clinical experience were invited to confirm (or correct) the labeling results. A total of 2664 images were excluded based on the following exclusion criteria: (1) poor image quality; (2) imaging artefacts; (3) unfocused scans; (4) presence of other disease phenotypes (e.g., retinal hemorrhage). No images were excluded based on age, sex, or race. Eventually, the remaining 36,231 images were included in the current study to build the intelligent system.

Each image was annotated with two labels: the classification label and the identification label. The classification labels constituted one of four degrees of ROP severity according to the requirements of clinical treatment [20]: "normal" (no abnormalities); "mild" (stage I or stage II, without plus disease; routine observation); "semi-urgent" (stage I or stage II, with plus disease; suggested referral); and "urgent" (stage III, stage IV, or stage $\mathrm{V}$, with or without plus disease; urgent referral for treatment). The identification labels were added to indicate ROP stages: "demarcation line," "ridge," "ridge with extra retinal fibrovascular involvement," "subtotal retinal detachment," and "total retinal detachment"; the identification labels were also added to indicate plus disease: "dilation and tortuosity of retinal vessels", based on the International Classification of Retinopathy of Prematurity system [21]. In addition, the lesion area was delineated with a box outline by the retinal experts. Representative ROP images are shown in Fig. 2. In addition, the experts labeled optic disc, fovea, and laser scar on the images to assist in diagnosis and monitoring of any therapeutic effects. 


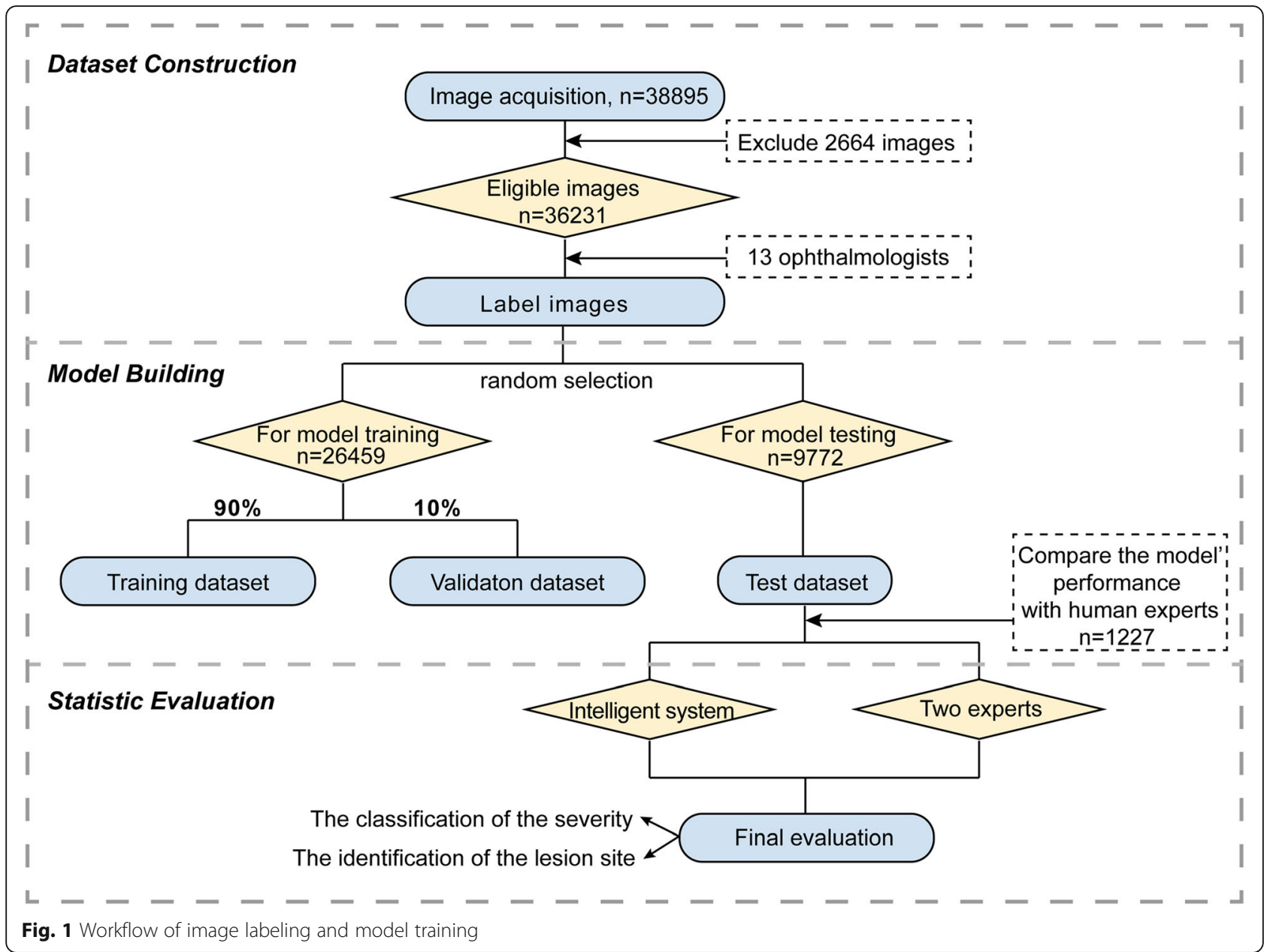

To account for image variation within our dataset, we used preprocessed versions of the original images and normalized the image before learning. The preprocessing steps consisted dataset augmentation followed by resizing. Data augmentation was a method that used image transformations across a sample dataset to increase image heterogeneity while preserving prognostic characteristics of the image itself. Since the fundus diagnosis primarily depended on the identification of major anatomical structures, regardless of orientation, we encoded rotational invariance into our predictions by randomly rotating images before propagating these images into our model. To preprocess images further before learning, we augmented the dataset by adding random noise and adjusted the image brightness. The large dataset improves the generalization of the model and reduces overfitting. Images were then downsized to a standard resolution of $224 \times 224$ pixels to fit the expected input size for algorithm training.

We randomly divided the obtained processed dataset into the training and test datasets. The training dataset was used to develop the learning model, while the test dataset was used to evaluate the model. Image numbers of each category in the training and test datasets are summarized in Table 1. During the training process, we used a conventional 10-fold cross-validation [22] method to evaluate and optimize our model. The sample was randomly partitioned into 10 complementary subsamples of equal size. Nine folds were selected as the training set and one was selected as the validation set over 10 iterations. Therefore, $90 \%$ of the data was used for training and $10 \%$ of the data was used for validation. In this context, all patients in the dataset participated in a validation, and each was predicted exactly once before the algorithms were ready to be tested.

\section{Development of the algorithm}

In this study, we used two deep CNNs: the 101-layer ResNet (classification network) and the Faster R-CNN (identification network). The model was built and trained with the Keras package in Python programming language (ver. 2.7.9, Python Software Foundation, Beaverton, US) using the TensorFlow backend (http://www.tensorflow. org). To improve the training speed, we utilized a ResNet- 


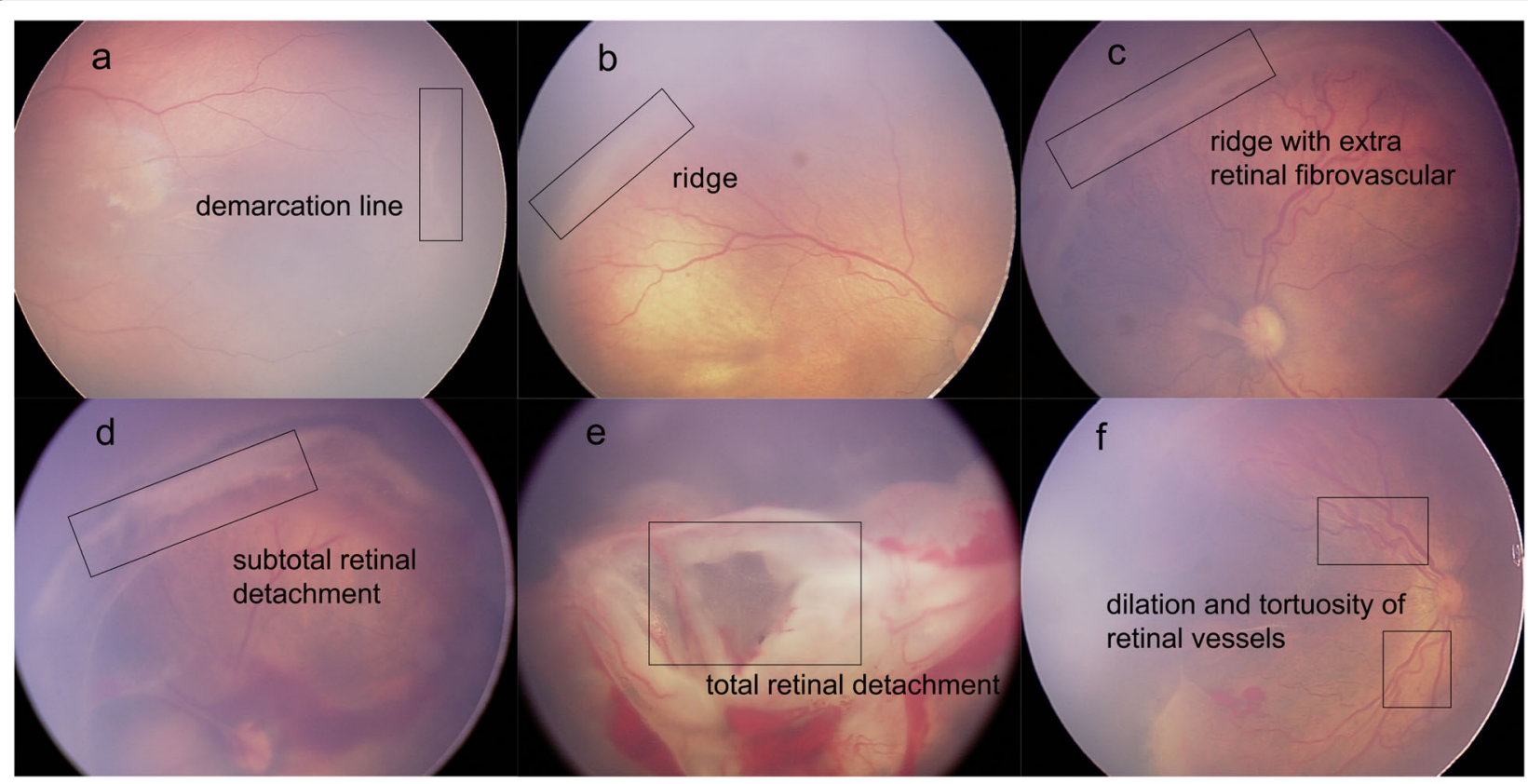

Fig. 2 Representative ROP images with annotations of five stages and the presence of plus disease. Box outlines in (a-f) indicate lesion sites. (a) Stage I: Demarcation line. (b) Stage II: Ridge. (c) Stage III: Ridge with extra retinal fibrovascular involvement. (d) Stage IV: Subtotal retinal detachment. (e) Stage V: Total retinal detachment. (f) Plus disease: Dilatation and tortuosity of retinal vessels

$101 \mathrm{CNN}$ architecture that was pre-trained on the ImageNet (http://www.image-net.org) database of 1.4 million images [23], and retrain it on our dataset using transfer learning, by which an algorithm can apply cumulative knowledge learned from other datasets to a new task [24]. The CNN consisted of multiple convolutional layers that learned local features of images and generated classifications. It included pooling layers (average pool and max pool) that merged semantically similar features into one feature, thereby reducing the dimensionality of the extracted features and fully connected layers to combine these features and provide a final probability value for the class. The original code of the study is available at https:// github.com/whu-eyelab/Rop_.
Recent studies have shown that network depth is beneficial to classification accuracy [25]. However, as the network gains greater depth, its performance becomes saturated and then begins to decrease rapidly [26]. The ResNet framework can correct this problem. Throughout the deep network, shortcut connections are added every three convolutional layers. These shortcut connections perform identity mapping without adding extra parameters or increasing the computational complexity, which enhances the ease of optimizing the network during the training process. Therefore, ResNet enables achievement of higher accuracy from deeper networks than from shallower networks when performing image classification tasks. The network was trained with a

Table 1 Number of images in the training and test datasets

\begin{tabular}{llll}
\hline Clinical features & Definition & Training dataset & Test dataset \\
\hline Stage I & Demarcation line & 1687 & 377 \\
Stage II & Ridge & 263 & 77 \\
Stage III & Ridge with extra retinal fibrovascular involvement & 179 & 48 \\
Stage IV & Subtotal retinal detachment & 45 & 13 \\
Stage V & Total retinal detachment & 10 & 4 \\
Plus disease & Dilation and tortuosity of retinal vessels & 2745 & 261 \\
Optic disc & - & 12,383 & 5278 \\
Fovea & - & 8455 & 3568 \\
Laser scar & - & 692 & 146 \\
Total & - & 26,459 & 9772 \\
\hline
\end{tabular}


learning rate of 0.0001 and the computation cost of $7.6 \times 10^{9}$ floating-point operations using the parameters presented in Fig. 3a.

Faster R-CNN is a high-performing object detection model, which was the winning entry of the Common Objects in Context (COCO) detection challenge [27]. Object detection involved recognition and classification of every object in an image as well as positioning each object within a bounding box. We configured Faster R$\mathrm{CNN}$ with a pretrained Inception-ResNet-v2 model provided by TensorFlow Object Detection application programming interface (Fig. 3b) to identify the stage of ROP and the presence of plus disease as well as to predict the objective boundaries of the lesion sites. The pretrained model had been trained on $\mathrm{COCO}$, which was a large image dataset designed for object detection [28]. During the training process, we applied the fine-tuning technique to transfer the connection weights from the pretrained model to our model and retrained the model to the present task. This model accepted an image as input and performed five main assessments: the region proposal network was used to identify object regions in an image; a classifier block of the outline box regressor and an object classifier were used to assess candidate boxes from the output of the region proposal network; region of interest pooling and fully connected layers were the final assessments. Eventually, the model outputs the bounding box of each target object as well as the corresponding category label.
We combined these two CNN networks as a system to process a large-scale ROP dataset; the system will eventually output the classification of ROP severity as well as the diagnosis of ROP stage and the presence of plus disease. The intelligent system ran a total of 120 training epochs (iterations) and the training stopped when the crossentropy loss function was minimized by stochastic gradient descent. Then, the model with the lowest loss (highest accuracy) was selected for use on the test dataset. TensorBoard chart was used to show the performance of model training and validation data (Fig. 4). All classifications produce convergence when training reaches the final layer. Figure 5 shows the overall working system.

\section{Statistical analysis}

To evaluate the performance of the intelligent system, three evaluation indicators were compared, including accuracy, sensitivity, specificity and F1-score. To further evaluate the performance of the system, 1227 fundus images captured during routine clinical ROP screening were used to compare the prediction accuracy of the system within the four-level classification relative to the diagnoses of two experienced human experts on retinal imaging. We also plotted the confusion matrices of the 101-layer ResNet and compared the locations of the lesions predicted by the intelligent system with those labeled by the experts. Statistical analyses were performed using GraphPad Prism software version 7.0 (GraphPad

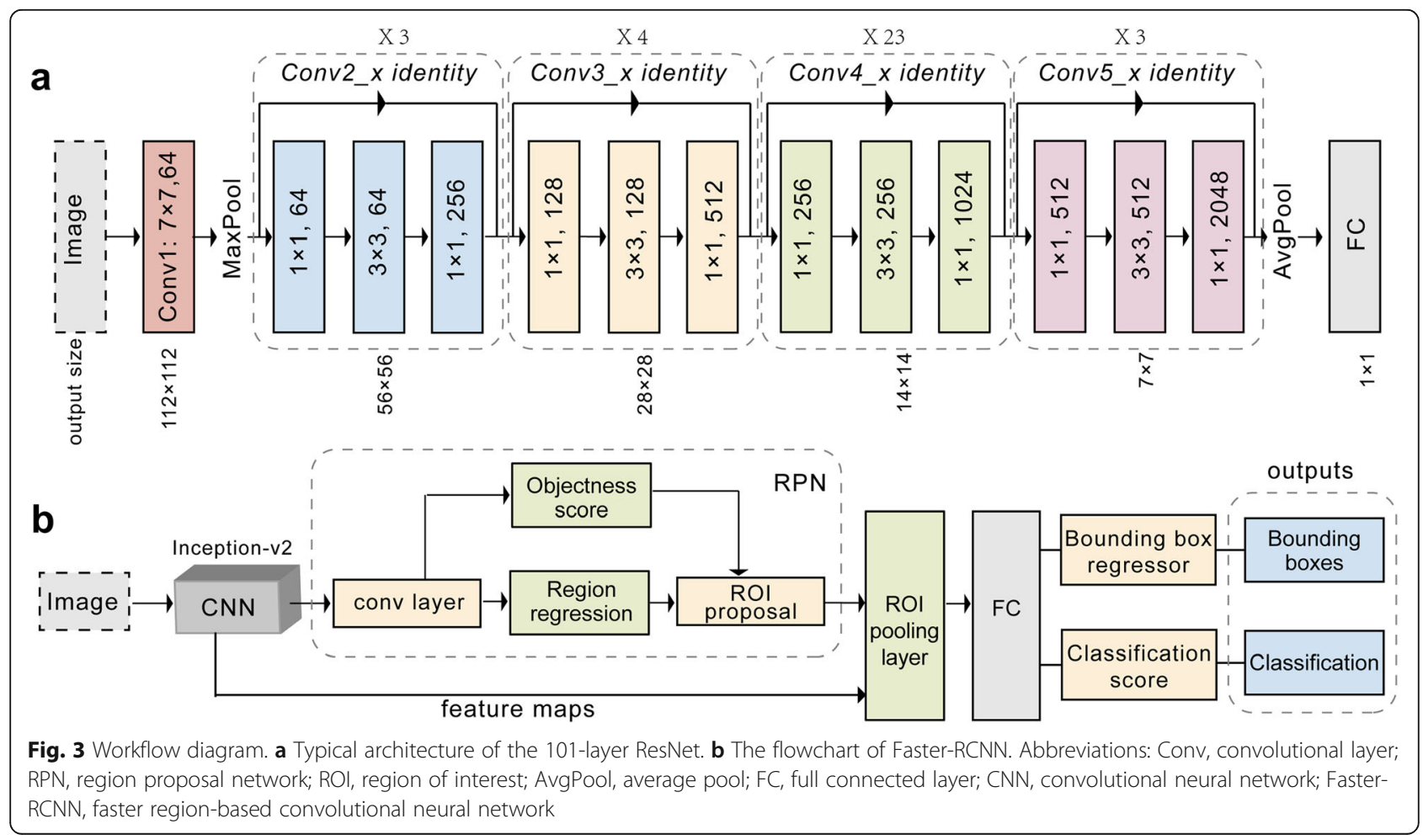




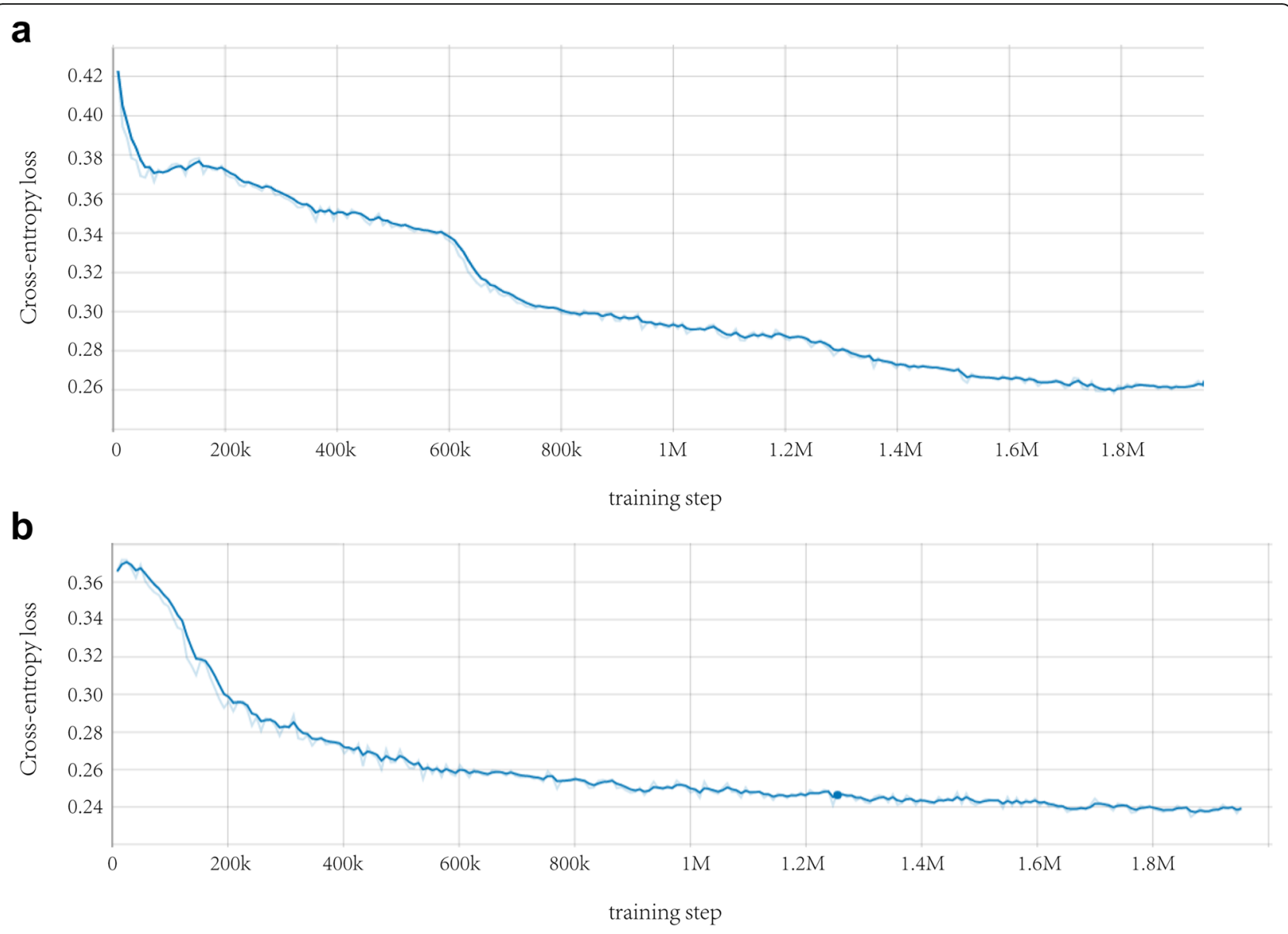

Fig. 4 Comparison between the cross-entropy loss function curve and the training step. (a) Performance of the training dataset; (b) Performance of the validation dataset

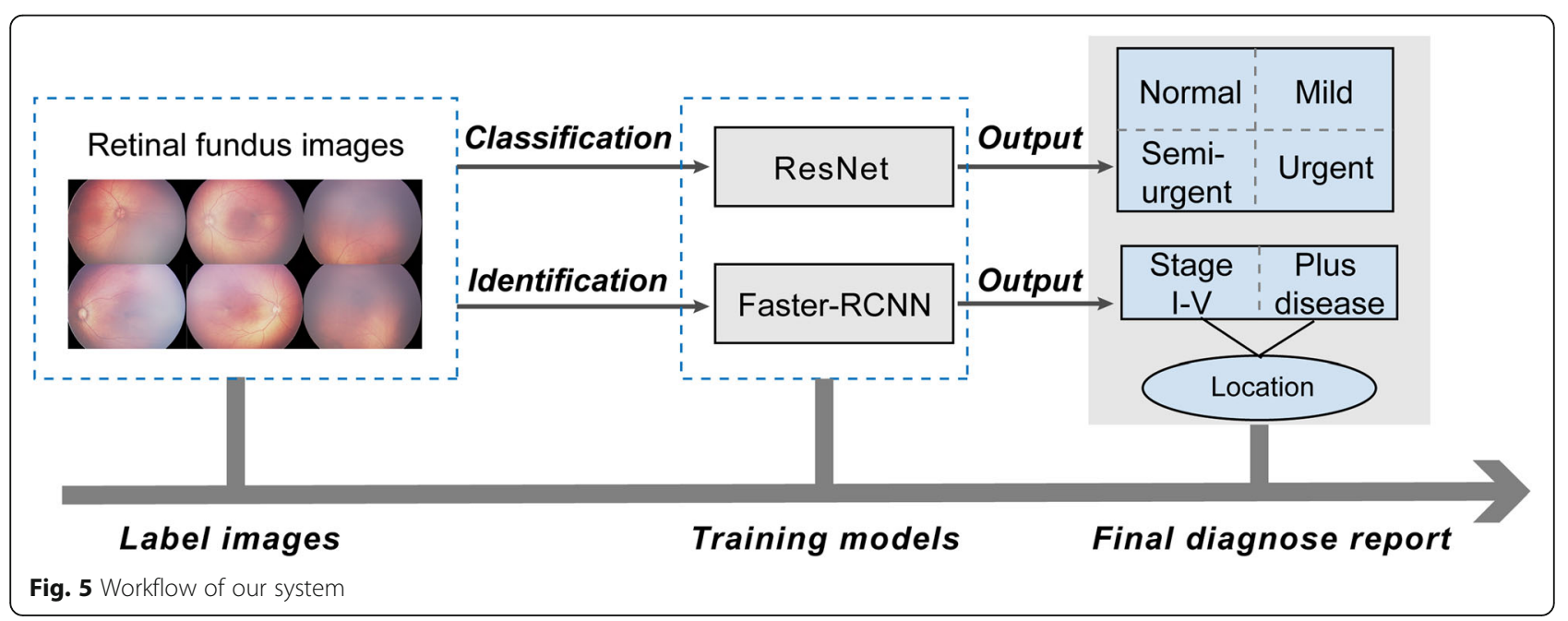


Inc., La Jolla, CA, USA) and IBM SPSS Statistics 19 (IBM Corp., Armonk, NY, USA).

\section{Results}

Our intelligent system was evaluated regarding its ability to discriminate the four-degree classification of ROP from fundus images; The results showed that the system can achieve an accuracy of 0.903 , a sensitivity of 0.778 with a specificity of 0.932 and a F1-score of 0.761 for grading the ROP cases as "normal," "mild," "semi-urgent," and "urgent" (Fig. 6).

We further compared the performance of the system with the results obtained by two experienced retinal experts. Expert 1 achieved an accuracy of 0.902, a sensitivity of 0.748 , a specificity of 0.934 and a F1-score of 0.743 ; expert 2 achieved an accuracy of 0.898 , a sensitivity of 0.659 , a specificity of 0.923 and a F1-score of 0.682 (Fig. 6). Table 2 shows the specific accuracies for each category obtained by the proposed system and the two retinal experts. The results showed that the system could correctly discriminate the four-degree classification with accuracies of $0.883,0.900,0.957$, and 0.870 , respectively.

Three confusion matrixes shown in Fig. 7 reveal the specific assignments of different predictions for each image. The rows provide the samples' true labels, while the columns present the predicted labels. Each diagonal element of the heatmap represents the percentage of images correctly classified in the corresponding category. Non-diagonal elements show the percentages of misclassified images and how they were misclassified. Misclassification cases and types were significantly fewer with the intelligent system than for human experts.
The accuracies of our system to identify the stage of ROP and the presence of plus disease were 0.957 and 0.896. Besides, it achieved an average F1-score of 0.78 in each category. Table 3 shows the specific accuracies, sensitivities and specificities of each category for the proposed system. The accuracies for discriminating stage I to stage V were $0.876,0.942,0.968,0.998$ and 0.999 , respectively.

Performance was also measured by evaluating whether the proposed outline boxes overlapped sufficiently with outline boxes that were provided as the gold standard. In the test phase, the re-trained model used test images as input then output the predicted category label and the outline box for each corresponding target object (Fig. 8).

\section{Discussion}

We developed a new automated feature-learning approach for ROP detection using DL methods. This provides a robust solution for ROP detection within a largescale annotated dataset, and the results showed high efficacy of the proposed model in providing objective and efficient ROP diagnosis without reliance on ophthalmologists for manual examination and grading of images. In addition to image classification, the system could accurately identify the stage of ROP and presence of plus disease, and could visualize abnormal regions, which are important for the clinical diagnosis of ROP.

By employing a transfer learning algorithm, the proposed system showed good performance for this application without the requirement for a highly specialized DL machine nor a novel database of millions of images. Key improvements are as follows: (1) a new dataset was constructed that is large and annotated with a new labeling

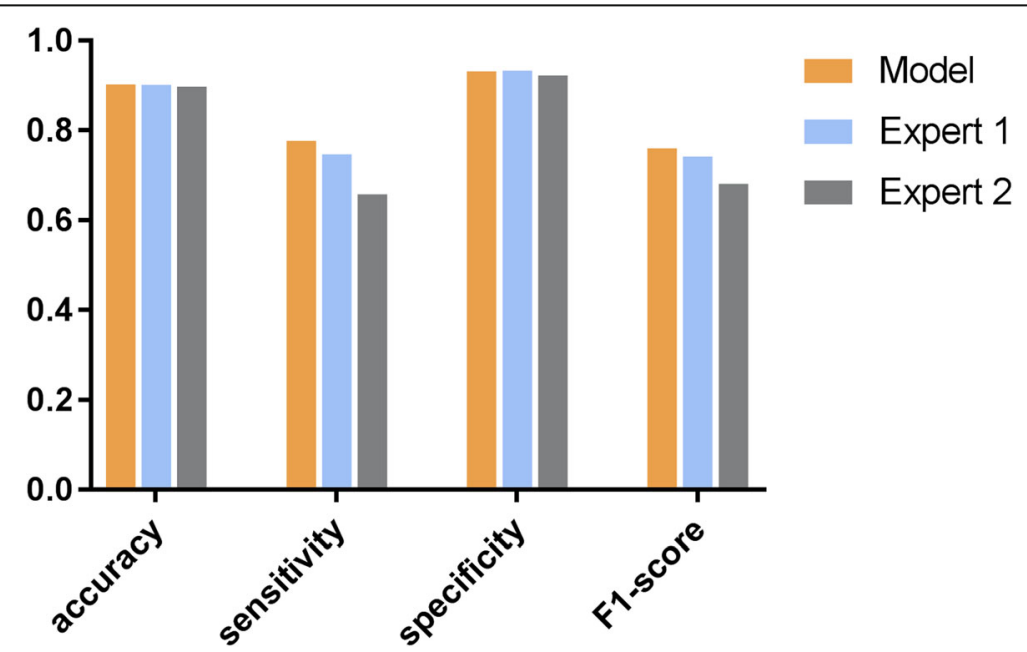

Fig. 6 Performances of the proposed system and the two human experts for the four-degree classification of the ROP severity. The proposed system demonstrated 0.903 accuracy, 0.778 sensitivity, 0.932 specificity and $0.761 \mathrm{~F} 1$-score for the four-degree classification task; Expert 1 achieved 0.902 accuracy, 0.748 sensitivity, 0.934 specificity and 0.743 F1-score, while expert 2 achieved 0.898 accuracy, 0.659 sensitivity, 0.923 specificity and 0.682 F1-score. Abbreviations: ROP, retinopathy of prematurity 
Table 2 The specific accuracies for each category by the intelligent system and the two retinal experts

\begin{tabular}{llll}
\hline Category & System & Expert 1 & Expert 2 \\
\hline Normal & 0.870 & 0.929 & 0.914 \\
Mild & 0.883 & 0.874 & 0.869 \\
Semi-urgent & 0.900 & 0.882 & 0.889 \\
Urgent & 0.957 & 0.923 & 0.920 \\
\hline
\end{tabular}

scheme combining more clinical features of ROP, aiding in reduction of the individual effect and avoiding overfitting of the algorithms to some specific feature; (2) two mainstream CNN models were applied as our classification and identification algorithms (101-layer ResNet and Faster-RCNN, respectively), which appeared to perform screening functions with proficiency comparable to or better than that of ROP experts; (3) the performance was optimized with a 10-fold cross-validation method that can increase the generalizability of the system.

Clinical studies have shown that zone I, any stage ROP with plus disease or zone I, stage III retinopathy without plus disease requires timely treatment to prevent blindness [29]. The most prominent advantage of our study is its attempt to identify the stage of ROP and presence of plus disease, along with disease severity; this functionality enables clinical review and verification of the automated diagnosis, rather than simply identifying the presence of ROP. Moreover, conventional deep neural networks (such as ResNet, AlexNet), provide only the image classification and associated labels without explicit definitions of features in clinical practice. Here, Faster-RCNN served as an object detection network that could recognize and classify object in an image and could position the object by using an outline box [27]; this enables ophthalmologists to inspect and visualize specific lesion regions. The algorithms developed in this study are advantageous in terms of the above properties when compared to other algorithms; the benefits also include consistent prediction and instantaneous reporting of results.
Previous studies of automated identification of ROP screening have shown encouraging results $[6,30,31]$. The majority of traditional methods for diagnosis of ROP are focused on the recognition of plus disease such as measuring the statistics of retinal vessels in the fundus [32]. For example, "ROPTool" and "i-ROP" systems were proposed to assist ophthalmologists in diagnosing plus disease [31, 33]. The ImageNet pre-trained GoogleNet was the first deep neural network to classify the presence of plus disease [34]. Although plus disease is an important clinical feature of ROP diagnosis by the International Classification of Retinopathy of Prematurity system defining treatment-requiring ROP, it is not sufficient to define ROP by itself only [21]. To the best of our knowledge, there has been minimal research focused on comparative analysis of image features that are most critical for diagnosis. In contrast to other studies, Wang J et al. developed a DL-based method and divided ROP into three grades with high sensitivity and specificity [35]. However, their system could only evaluate the severity of ROP; it could not identify finer details, such as the stage of ROP or presence of plus disease. Additionally, the numbers of the images in different datasets are insufficient to develop robust DL models that can deliver satisfactory performance [36]. An overview of previous studies using AI methods for ROP diagnosis are listed in Table 4, including a comparison of the dataset, diagnostic model and their applications in ROP.

Some limitations of this study include: (1) limited number of ROP stage $\mathrm{V}$ fundus images in our dataset, which may have biased the performance of the model; (2) the fundus images in our study were collected from a single clinical site with consistent device settings and population characteristics, which might have reduced data diversity and affected the generalization ability of the algorithm; (3) our system struggled to differentiate between normal and very early cases of ROP in the dataset, such that it missed cases with subtle demarcation lines; (4) although we used a cross-validation method to maximize generalizability to other datasets, an important

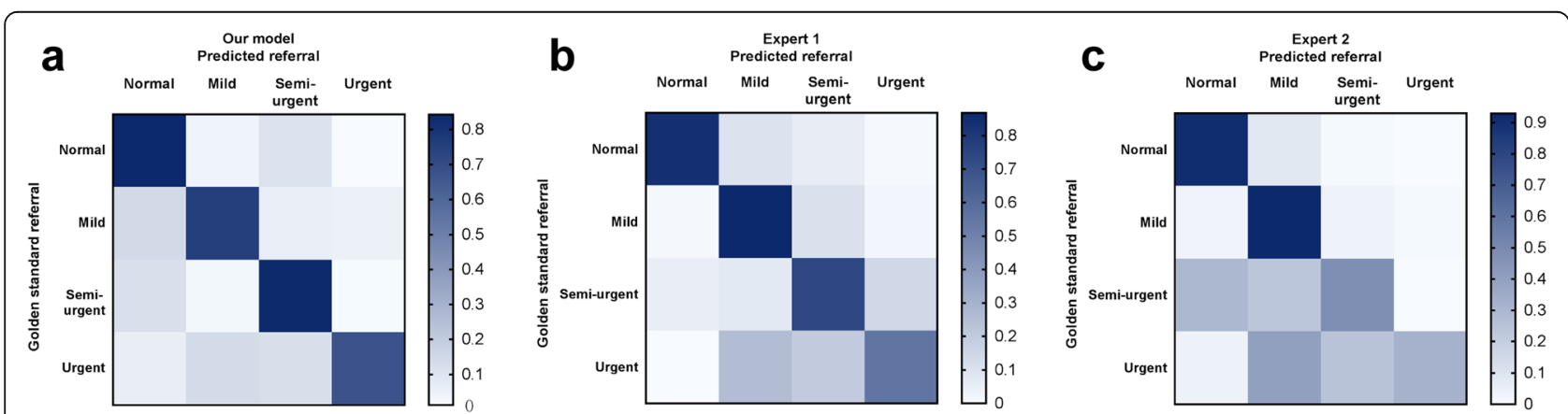

Fig. 7 Three confusion matrixes for the intelligent system and the two best retina experts' predictions in the four-degree classification task. (a) Confusion matrix of the proposed system; (b) Confusion matrix of expert 1; (c) Confusion matrix of expert 2 
Table 3 The accuracies, sensitivities, specificities and F1-scores for each category by the proposed system

\begin{tabular}{|c|c|c|c|c|c|}
\hline Definition & Clinical features & Accuracy & Sensitivity & Specificity & F1-score \\
\hline Stage I & Demarcation line & 0.876 & 0.765 & 0.883 & 0.81 \\
\hline Stage II & Ridge & 0.942 & 0.550 & 0.973 & 0.52 \\
\hline Stage III & Ridge with extra retinal fibrovascular & 0.968 & 0.473 & 0.975 & 0.51 \\
\hline Stage IV & Subtotal retinal detachment & 0.998 & 0.867 & 0.998 & 0.93 \\
\hline Stage V & Total retinal detachment & 0.999 & 0.800 & 0.999 & 0.89 \\
\hline Plus disease & Dilation and tortuosity of retinal vessels & 0.896 & 0.713 & 0.907 & 0.78 \\
\hline Optic disc & & 0.954 & 0.945 & 0.917 & 0.96 \\
\hline Fovea & & 0.781 & 0.744 & 0.840 & 0.72 \\
\hline Laser scars & & 0.974 & 0.908 & 0.988 & 0.89 \\
\hline
\end{tabular}

continuation to this study will be to achieve validation using completely separate images. Notably, premature infants who were diagnosed with avascular retina but no characteristics of ROP for the first screening time, needed to be followed up every $2-3$ weeks until the retina was fully vascularized.

In future studies, larger datasets of severe ROP images are needed to validate and optimize our system in the clinical setting. Moreover, further testing and optimization of the sensitivity metric may be necessary to ensure a minimum false-negative rate. Additionally, multimodal clinical metadata should be included in the AI diagnosis of ROP, such as birth weight, patient history, gestational age, and other clinical data that may influence the risk of retinopathy. Datasets from multiple clinical centers and larger patient cohorts are needed in subsequent studies to further validate this intelligent system and enable it to serve as a practical intelligent tool for real-world clinical use.

\section{Conclusions}

Overall, our DL-based system showed the potential for automated detection of ROP and differentiation of fourlevel classification fundus images with high accuracy and specificity. The performance of the system was equal to or better than that of retinal experts, suggesting that this system can be used to assist in clinical decisions; this is

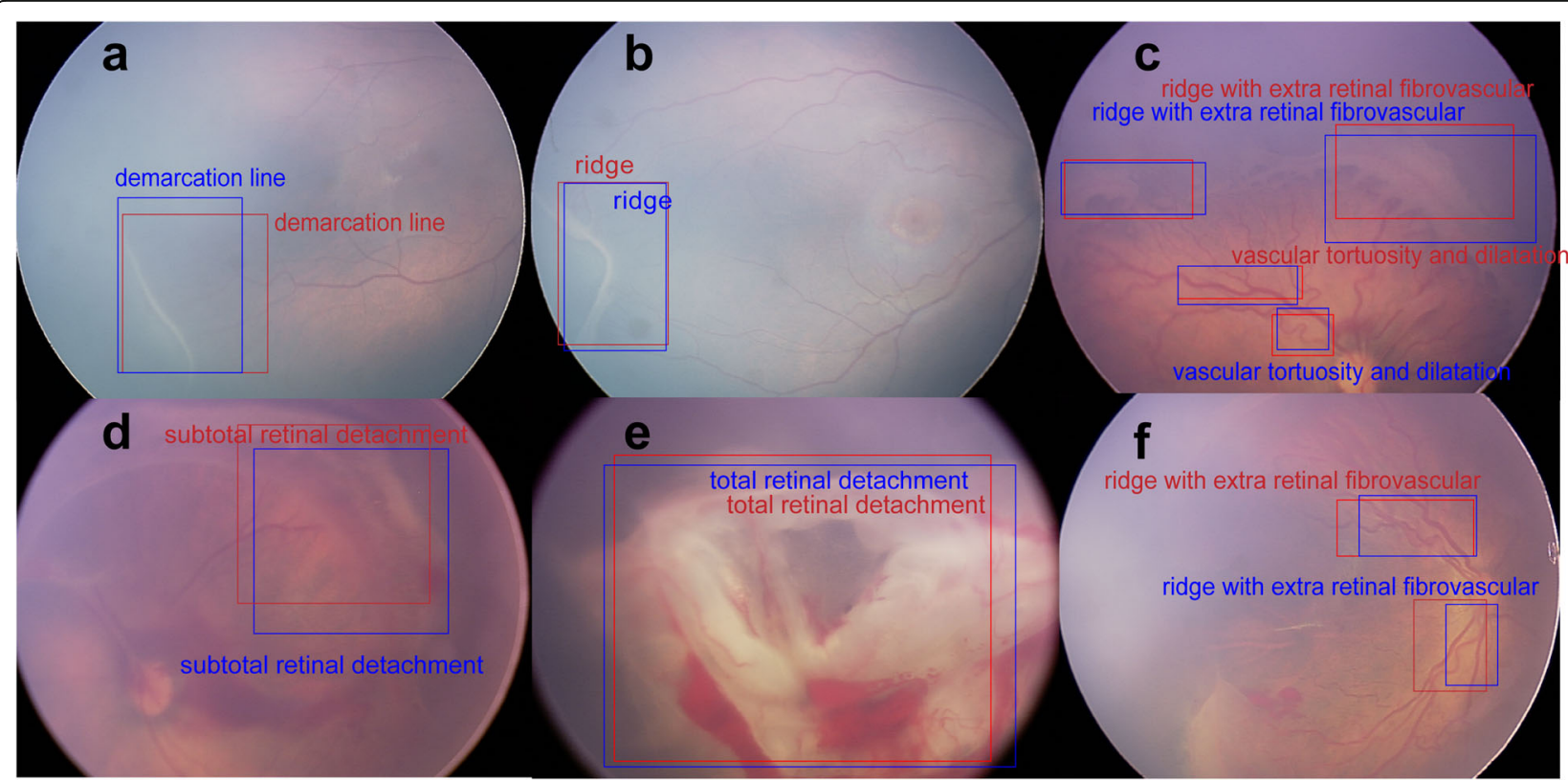

Fig. 8 Representative images of system-predicted lesion locations compared with those predicted by experts. Box outlines in (a-f) indicate lesion sites. The boxes in red are the lesion locations annotated by retinal experts (gold standard annotations), and the boxes in blue are the lesion locations predicted by the Faster-RCNN. Abbreviation: Faster-RCNN, faster region-based convolutional neural network 


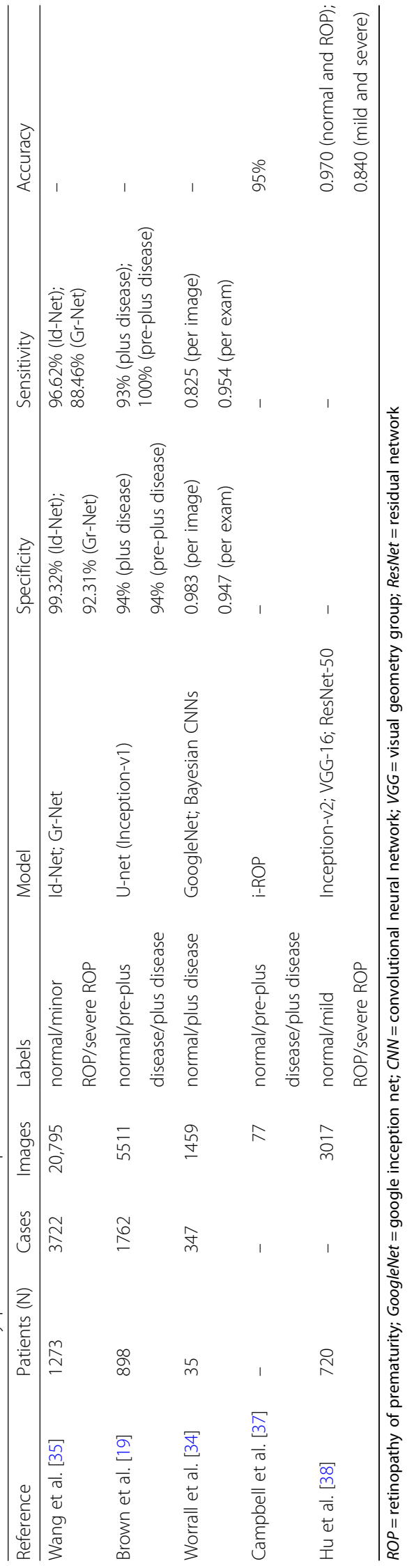


an initial step toward clinical translation of this method. Given the increasing burden of ROP on the healthcare system, the implementation of our algorithm is likely to be important in supporting decisions for patient management and primary care-based screening approaches for ROP in the general population.

\section{Abbreviations}

ROP: Retinopathy of prematurity; Faster-RCNN: Faster region-based convolutional neural network; Al: Artificial intelligence; DL: Deep learning; CNN: Convolutional neural network; Conv: Convolutional layer; RPN: Region proposal network; ROI: Region of interest; AvgPool: Average pool; FC: Full connected layer; GoogleNet: Google inception net; VGG: Visual geometry group; ResNet: Residual network

\section{Acknowledgements}

We thank Mr. Yu Yue and Mr. Bin Wang for their help and valuable suggestions in the development of the deep learning algorithm for this research. We acknowledge Dr. Xiao-Ling Wang for her help in collecting the fundus images.

\section{Authors' contributions}

YT contributed to study design, data analysis and interpretation and drafted the manuscript. WL contributed to data collection and manuscript discussion. QQD and CZC contributed to data collection and designed the protocol. YS conceived the study, reviewed, and revised the manuscript. All authors read and approved the final manuscript.

\section{Funding}

This work was supported by: The National Key R\&D Program of China (Grant No. 2017YFE0103400); The National Nature Science Foundation of China (Grant No. 81470628)

\section{Availability of data and materials}

The dataset used and analyzed during the current study are available from the corresponding author on reasonable request.

\section{Ethics approval and consent to participate}

This study was approved by the institutional review board of Renmin Hospital of Wuhan University (ID: WDRY2019-K032)

\section{Consent for publication}

Not applicable.

\section{Competing interests}

The authors declare that they have no competing interests.

Received: 20 January 2020 Accepted: 2 July 2020

Published online: 01 August 2020

\section{References}

1. Sommer A, Taylor HR, Ravilla TD, West S, Lietman TM, Keenan JD, et al. Challenges of ophthalmic care in the developing world. JAMA Ophthalmol. 2014;132(5):640-4.

2. Stahl A, Goepel W. Screening and treatment in retinopathy of prematurity. Deutsches Arzteblatt Int. 2015;112(43):730-5

3. Blencowe H, Lawn JE, Vazquez T, Fielder A, Gilbert C. Preterm-associated visual impairment and estimates of retinopathy of prematurity at regional and global levels for 2010. Pediatr Res. 2013;74(Suppl 1):35-49.

4. Tasman WS. Revised indications for the treatment of retinopathy of prematurity results of the early treatment for retinopathy of prematurity randomized trial. Evidence-Based Eye Care. 2004;5(3):156-7.

5. Chiang MF, Thyparampil PJ, Rabinowitz D. Interexpert agreement in the identification of macular location in infants at risk for retinopathy of prematurity. Arch Ophthalmol. 2010;128(9):1153-9.

6. Bolón-Canedo V, Ataer-Cansizoglu E, Erdogmus D, Kalpathy-Cramer J, Fontenla-Romero O, Alonso-Betanzos A, et al. Dealing with inter-expert variability in retinopathy of prematurity: a machine learning approach. Comput Methods Prog Biomed. 2015;122(1):1-15.

7. LeCun Y, Bengio Y, Hinton G. Deep learning. Nature. 2015;521(7553):436-44.
8. Esteva A, Kuprel B, Novoa RA, Ko J, Swetter SM, Blau HM, et al. Dermatologist-level classification of skin cancer with deep neural networks. Nature. 2017;542(7639):115-8.

9. van Ginneken B. Fifty years of computer analysis in chest imaging: rule-based, machine learning, deep learning. Radiol Phys Technol. 2017;10(1):23-32.

10. Ertosun MG, Rubin DL. Automated grading of gliomas using deep learning in digital pathology images: a modular approach with ensemble of convolutional neural networks. AMIA Annu Symp Proc. 2015;2015:1899-908.

11. Bejnordi BE, Zuidhof G, Balkenhol M, Hermsen M, Bult P, van Ginneken B, et al. Context-aware stacked convolutional neural networks for classification of breast carcinomas in whole-slide histopathology images. J Med Imaging (Bellingham). 2017:4(4):044504.

12. Schmidt-Erfurth U, Sadeghipour A, Gerendas BS, Waldstein SM, Bogunović H. Artificial intelligence in retina. Prog Retin Eye Res. 2018;67:1-29.

13. Ting DSW, Peng L, Varadarajan AV, Keane PA, Burlina PM, Chiang MF, et al. Deep learning in ophthalmology: the technical and clinical considerations. Prog Retin Eye Res. 2019;72:100759.

14. Grzybowski A, Brona P, Lim G, Ruamviboonsuk P, Tan GSW, Abramoff M, et al. Artificial intelligence for diabetic retinopathy screening: a review. Eye (Lond). 2020;34(3):451-60.

15. Devalla SK, Liang Z, Pham TH, Boote C, Strouthidis NG, Thiery AH, et al. Glaucoma management in the era of artificial intelligence. Br J Ophthalmol. 2020;104(3):301-11

16. Reid JE, Eaton E. Artificial intelligence for pediatric ophthalmology. Curr Opin Ophthalmol. 2019;30(5):337-46.

17. Wittenberg LA, Jonsson NJ, Chan RV, Chiang MF. Computer-based image analysis for plus disease diagnosis in retinopathy of prematurity. J Pediatr Ophthalmol Strabismus. 2012;49(1):11-9.

18. Gelman R, Jiang L, Du YE, Martinez-Perez ME, Flynn JT, Chiang MF. Plus disease in retinopathy of prematurity: pilot study of computer-based and expert diagnosis. J AAPOS. 2007;11(6):532-40.

19. Brown JM, Campbell JP, Beers A, Chang K, Ostmo S, Chan RVP, et al. Automated diagnosis of plus disease in retinopathy of prematurity using deep convolutional neural networks. JAMA Ophthalmol. 2018;136(7):803-10.

20. Early Treatment for Retinopathy of Prematurity Cooperative Group. Revised indications for the treatment of retinopathy of prematurity: results of the early treatment for retinopathy of prematurity randomized trial. Arch Ophthalmol. 2003;121(12):1684-94.

21. International Committee for the Classification of Retinopathy of Prematurity. The international classification of retinopathy of prematurity revisited. Arch Ophthalmol. 2005;123(7):991-9.

22. Cherkassky $\mathrm{V}$. The nature of statistical learning theory . IEEE Trans Neural Netw. 1997:8(6):1564.

23. He K, Zhang $X$, Ren S, Sun J. Deep residual learning for image recognition. In: 2016 IEEE conference on computer vision and pattern recognition (CVPR); 2016

24. Pan SJ, Yang Q. A survey on transfer learning. IEEE Trans Knowl Data Eng. 2010;22(10):1345-59

25. Szegedy C, Wei L, Yangqing J, Sermanet P, Reed S, Anquelov D, et al. Going deeper with convolutions. In: 2015 IEEE conference on computer vision and pattern recognition (CVPR); 2015.

26. He K, Sun J. Convolutional neural networks at constrained time cost. In: 2015 IEEE conference on computer vision and pattern recognition (CVPR); 2015.

27. Ren S, He K, Girshick R, Sun J. Faster R-CNN: towards real-time object detection with region proposal networks. IEEE Trans Pattern Anal Mach Intell. 2017;39(6):1137-49.

28. Lin T-Y, Maire M, Belongie S, Hays J, Perona P, Ramanan D, et al. Microsoft COCO: common objects in context. In: Computer Vision - ECCV 2014; 2014. p. 740-55.

29. Fielder AR. Preliminary results of treatment of eyes with high-risk prethreshold retinopathy of prematurity in the early treatment for retinopathy of prematurity randomized trial. Arch Ophthalmol. 2003;121(12):1769-71.

30. Ataer-Cansizoglu E, Kalpathy-Cramer J, You S, Keck K, Erdogmus D, Chiang MF. Analysis of underlying causes of inter-expert disagreement in retinopathy of prematurity diagnosis. Methods Inf Med. 2015;54(1):93-102.

31. Ataer-Cansizoglu E, Bolon-Canedo V, Campbell JP, Bozkurt A, Erdogmus D, Kalpathy-Cramer J, et al. Computer-based image analysis for plus disease diagnosis in retinopathy of prematurity: performance of the "i-ROP" system and image features associated with expert diagnosis. Transl Vis Sci Technol. 2015;4(6):5.

32. Aslam T, Fleck B, Patton N, Trucco M, Azegrouz H. Digital image analysis of plus disease in retinopathy of prematurity. Acta Ophthalmol. 2009;87(4):368-77. 
33. Wallace DK, Zhao Z, Freedman SF. A pilot study using "ROPtool" to quantify plus disease in retinopathy of prematurity. J AAPOS. 2007;11(4):381-7.

34. Worrall DE, Wilson CM, Brostow GJ. Automated retinopathy of prematurity case detection with convolutional neural networks. In: Deep learning and data labeling for medical applications; 2016. p. 68-76.

35. Wang J, Ju R, Chen Y, Zhang L, Hu J, Wu Y, et al. Automated retinopathy of prematurity screening using deep neural networks. EBioMedicine. 2018;35:361-8.

36. Silver D, Huang A, Maddison CJ, Guez A, Sifre L, van den Driessche G, et al. Mastering the game of Go with deep neural networks and tree search. Nature. 2016;529(7587):484-9.

37. Campbell JP, Ataer-Cansizoglu E, Bolon-Canedo V, Bozkurt A, Erdogmus D, Kalpathy-Cramer J, et al. Expert diagnosis of plus disease in retinopathy of prematurity from computer-based image analysis. JAMA Ophthalmol. 2016; 134(6):651-7.

38. Hu J, Chen Y, Zhong J, Ju R, Yi Z. Automated analysis for retinopathy of prematurity by deep neural networks. IEEE Trans Med Imaging. 2019;38(1):269-79.

Ready to submit your research? Choose BMC and benefit from:

- fast, convenient online submission

- thorough peer review by experienced researchers in your field

- rapid publication on acceptance

- support for research data, including large and complex data types

- gold Open Access which fosters wider collaboration and increased citations

- maximum visibility for your research: over $100 \mathrm{M}$ website views per year

At BMC, research is always in progress.

Learn more biomedcentral.com/submissions 